\title{
Adrenal insufficiency in immunochemotherapy for small-cell lung cancer with ectopic ACTH syndrome
}

\author{
Hiroki Nakajima1, Yasuhiro Niida1, Eriko Hamada², Kuwata Hirohito1, Masahide Ota², Sadanori Okada1, \\ Takako Mohri1, Yukako Kurematsu1, Shigeto Hontsu², Shigeo Muro² and \\ Yutaka Takahashi1 \\ 'Department of Diabetes and Endocrinology and 2Department of Respiratory Medicine, Nara Medical University, \\ Kashihara, Japan
}

Correspondence should be addressed to $Y$ Takahashi Email

takahash@naramed-u.ac.jp

\section{Summary}

Ectopic ACTH (adrenocorticotrophic hormone) syndrome (EAS) is rarely associated with small-cell lung cancer (SCLC). Although chemotherapy is initially effective for SCLC, complicated EAS scarcely improves. Recently, immune checkpoint inhibitors have been used to treat SCLC. Atezolizumab plus chemotherapy for SCLC improved progression-free survival compared to conventional chemotherapy. However, little has been reported on the efficacy of the combination therapy for SCLC with EAS. We report a 72-year-old male who presented with 4-week history of leg oedema, proximal myopathy, weight loss, and worsened symptoms of diabetes and hypertension. Laboratory findings revealed hypokalaemia, increased plasma ACTH, and serum cortisol levels. Cortisol levels were not suppressed by the high-dose dexamethasone test. Chest and abdominal CT revealed a right lower lobe tumour with multiple metastases on the hilar lymph nodes, liver, lumbar spine, and bilateral enlarged adrenal glands. The patient was diagnosed with stage 4B SCLC with EAS. Hypercortisolaemia was then treated with metyrapone and atezolizumab plus chemotherapy, which was started for SCLC. After 10 days, the tumour shrank noticeably, and the ACTH level drastically decreased concomitantly with low cortisol levels with symptoms of fever, appetite loss, and general fatigue. Hydrocortisone treatment was initiated, and the symptoms resolved immediately. We describe a case of SCLC with EAS treated with atezolizumab plus chemotherapy, presenting with adrenal insufficiency. Close observation is required for patients with adrenal insufficiency receiving atezolizumab plus chemotherapy because of its stronger effect. Furthermore, advances in cancer therapy and care for endocrine paraneoplastic syndrome needs to be adapted.

\section{Learning points:}

- The immune checkpoint inhibitor atezolizumab has recently been approved for the treatment of small-cell lung cancer (SCLC).

- Approximately 1-6\% of tumour ectopically produce ACTH and cause ectopic ACTH syndrome (EAS) as an endocrine paraneoplastic syndrome.

- The use of combined chemotherapy and atezolizumab in the ectopic ACTH syndrome secondary to small-cell lung cancer may cause a precipitous fall in circulating ACTH/cortisol, resulting in symptomatic adrenal insufficiency

- The advances in cancer therapy and treatment for endocrine paraneoplastic syndrome need to be adapted. 


\section{Background}

Small-cell lung cancer (SCLC) has been treated with chemotherapy as it is sensitive to cytotoxic agents at least for several months (1). SCLC originates from neuroendocrine cells and is classified as a neuroendocrine tumour (NET), (1) and $1--6 \%$ of tumour ectopically produce ACTH (adrenocorticotrophic hormone) that cause ectopic ACTH syndrome (EAS) (2). In this condition, tumour generally shows chemotherapy resistance and frequently some serious complications (2), resulting in poor prognosis than that without EAS; its median survival has reportedly been 6.6 months (2). First-line chemotherapy with atezolizumab, an immune checkpoint inhibitor (ICI) for extensive-stage SCLC, has shown a better prognosis than the conventional chemotherapy (3), and atezolizumab for SCLC was newly approved by the Food and Drug Administration (FDA) in March 2019. However, there have been few reports regarding the effect of chemotherapy with atezolizumab for SCLC with EAS. Here, we report a case of SCLC with EAS treated with chemotherapy with atezolizumab that demonstrates the clinical course of Cushing's syndrome.

\section{Case presentation}

A 72-year-old male who presented with a 4-week history of leg oedema, proximal myopathy, and weight loss was referred to our hospital. The patient has a history of Graves' disease and diabetes mellitus and hypertension, which rapidly worsened for 2 months before the visit. In addition, he had smoked 20 cigarettes daily for 51 years. He was treated with metformin, ipragliflozin, sitagliptin, metformin, pravastatin, telmisartan, amlodipine, mexiletine, carvedilol, and thiamazole.

\section{Investigation}

During administration, the patient's blood pressure was $158 / 60 \mathrm{mmHg}$, and he showed no classical Cushingoid appearance. Laboratory findings were as follows: white blood cell count: $1.39 \times 10^{9} / \mathrm{L}$ (neutrophil: $1.30 \times 10^{9} / \mathrm{L}$, eosinophil: $0.00 \times 10^{9} / \mathrm{L}$ ), blood glucose: $22.1 \mathrm{mmol} / \mathrm{L}$, haemoglobin A1c: 11.0\%, serum sodium: $139 \mathrm{mmol} / \mathrm{L}$, serum potassium: $3.5 \mathrm{mmol} / \mathrm{L}, \mathrm{LH}:<0.30 \mathrm{IU} / \mathrm{L}, \mathrm{FSH}: 0.35$ $\mathrm{IU} / \mathrm{L}$, prolactin: $199.3 \mu \mathrm{IU} / \mathrm{mL}$, testosterone: $7.6 \mathrm{nmol} / \mathrm{L}$, insulin-like growth factor 1: $26 \mathrm{ng} / \mathrm{mL}$, serum growth hormone: $0.32 \mathrm{ng} / \mathrm{mL}$, DHEA-S: $17.5 \mu \mathrm{mol} / \mathrm{L}$, TSH: 0.63 $\mathrm{mIU} / \mathrm{L}$, freeT3: $1.23 \mathrm{pmol} / \mathrm{L}$, and freeT4: $5.53 \mathrm{pmol} / \mathrm{L}$. Tumour markers for SCLC were markedly elevated; neuron- specific enolase (NSE) and progastrin-releasing peptide (Pro-GRP) were $19.4 \mathrm{ng} / \mathrm{mL}$ and $11400 \mathrm{pg} / \mathrm{mL}$, respectively. Endocrinological examination demonstrated that plasma ACTH levels at 10:00, 16:00, and 23:00 h were 153.3, 126.1, and $203.1 \mathrm{pmol} / \mathrm{L}$, respectively. Serum cortisol levels were 2543.8, 2684.5, and $2176.9 \mathrm{nmol} / \mathrm{L}$, respectively. High-dose dexamethasone $(8 \mathrm{mg}$ ) did not suppress serum cortisol levels. The brain MRI showed no obvious findings in the pituitary gland. In addition, chest and abdominal contrastenhanced CT scans revealed a $25 \mathrm{~mm}$ diameter nodule with an irregular surface in the right lower lobe (Fig. 1A), swelling of the right hilar lymph nodes, thickening of the pleura, multiple liver tumours (Fig. 1B), osteosclerotic lesions in the lumbar spine, and bilateral enlarged adrenal glands (Fig. 1C). Although biopsy was performed by bronchoscopy, only necrotic tissue was obtained. Collectively, we diagnosed SCLC with EAS.

\section{Treatment}

After the diagnosis of EAS, metyrapone was administered, and the dose was increased according to the serum cortisol levels (Fig. 2). The patient received chemotherapy consisting of four cycles of carboplatin at a dose of 450 $\mathrm{mg} /$ body on day 1 of each cycle, etoposide at a dose of 160 $\mathrm{mg} /$ body $\left(100 \mathrm{mg} / \mathrm{m}^{2}\right)$ on days 1 through 3 of each cycle, and atezolizumab at a dose of $1200 \mathrm{mg} /$ body on day 1 of each cycle in the induction phase. Only atezolizumab was administered at a dose of $1200 \mathrm{mg} /$ body on day 1 of each cycle in the maintenance phase.

\section{Outcome and follow-up}

On day 1, an initial dose of metyrapone $750 \mathrm{mg}$ /day was given and then titrated to $3000 \mathrm{mg}$ divided into three times per day. After the first cycle of chemotherapy, the tumour rapidly shrank, and ACTH levels drastically decreased with a concomitant decrease in cortisol levels (Fig. 2). Ten days after the initiation of chemotherapy, the patient complained of fever, appetite loss, and general fatigue. At that time, plasma ACTH levels were 18.0 $\mathrm{pmol} / \mathrm{L}$, and serum cortisol levels were $182.1 \mathrm{nmol} / \mathrm{L}$. Therefore, hydrocortisone $20 \mathrm{mg}$ per day (divided into three doses at 10, 5, and $5 \mathrm{mg}$ ) was initiated. However, his symptoms did not improve satisfactorily, probably due to stress; therefore, the dose was increased to $40 \mathrm{mg}$, and the symptoms rapidly resolved. After a month, the patient was moved to the maintenance dose of $15 \mathrm{mg}$. Moreover, 5 weeks after the initiation of chemotherapy, plasma ACTH levels were within a normal range (11.4 pmol/L), 

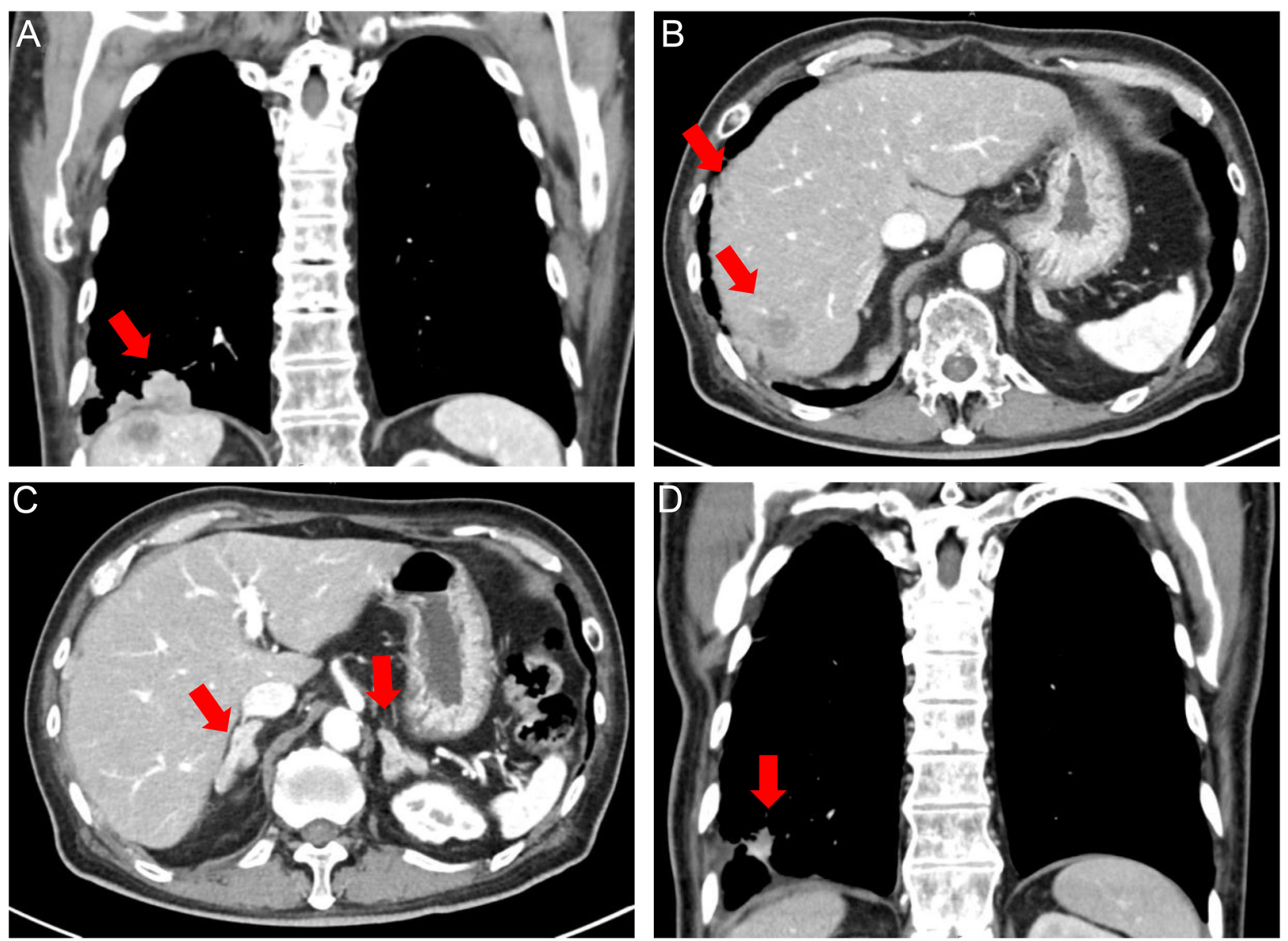

\section{Figure 1}

(A) Chest CT scan at the diagnosis shows 25-mm diameter nodule with an irregular surface in the right lower lobe with pleural thickening. (B) CT scan shows multiple liver metastases. (C) Bilateral adrenal hyperplasia. (D) Tumour shows visible shrinkage after four cycles of chemotherapy (the coronal plane at the maximum diameter of tumour).

and serum cortisol levels were $237.3 \mathrm{nmol} / \mathrm{L}$; therefore, metyrapone was discontinued, and hydrocortisone replacement therapy was continued. Additionally, we performed a brain MRI when adrenal insufficiency was observed to rule out metastatic brain lesions and revealed no lesions, including the pituitary. After four cycles of chemotherapy, the tumour shrank (Fig. 1D), and Pro-GRP and NSE levels markedly decreased $(316 \mathrm{pg} / \mathrm{mL}$ and 12.0 $\mathrm{ng} / \mathrm{mL}$, respectively).

Treatment with atezolizumab was effective for a while ( day 151); however, thereafter the disease progressed. On day 201, serum ACTH levels increased significantly (163.1 pmol/L) and metyrapone was started again. Simultaneously, second-line chemotherapy (amrubicin) was initiated. However, the patient refused to continue further chemotherapy because of its side effects. Eleven months after the diagnosis, the patient died.

\section{Discussion}

We described a case of SCLC with EAS, demonstrating that atezolizumab plus conventional chemotherapy showed a drastic effect and a transient but complete remission of EAS; however, the patient manifested an adrenal insufficiency, and a prolonged adrenal replacement therapy was needed. In practice, although uncontrollable hypercortisolaemia, despite treatment with steroidogenesis inhibitors such as metyrapone, was frequently experienced, adrenal insufficiency during the clinical course was rare (2). For example, it has been reported that among seven patients, conventional chemotherapy did not reduce plasma ACTH levels in two patients. Although five patients showed a transient reduction of ACTH levels reduced concomitantly with decreased tumour size and a duration of 1.5-3 months to reach normal ACTH levels, there have been no reports 


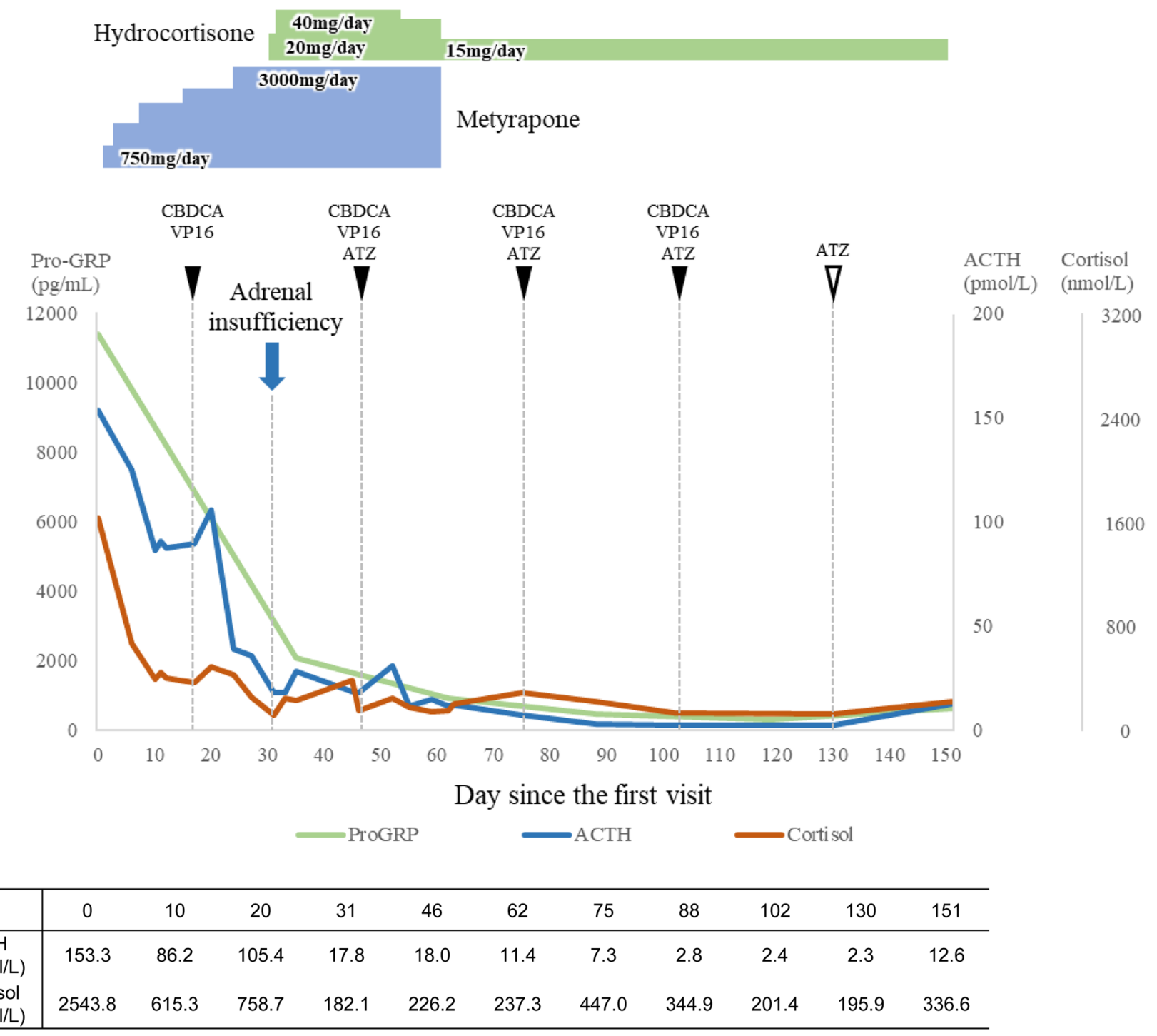

Figure 2

Clinical course of tumour marker pro-GRP, plasma ACTH, and serum cortisol levels during the treatment. Immediately after the first cycle of the chemotherapy, the patient manifested adrenal insufficiency, and subsequently, metyrapone for the treatment of hypercortisolaemia was discontinued. CBDCA denotes carboplatin, VP16 denotes etoposide, and ATZ denotes atezolizumab.

regarding adrenal insufficiency during chemotherapy to the best of our knowledge $(1,4,5,6,7,8)$.

In our patient, atezolizumab plus conventional chemotherapy was administered, and the effect on the tumour and plasma ACTH levels was rapid and significant. The tumour substantially shrank, and ACTH levels normalised within 5 weeks. Furthermore, the patient manifested adrenal insufficiency; therefore, we began hydrocortisone replacement therapy and discontinued metyrapone. The clinical course remarkably reflects the high efficiency of the new chemotherapy regimen, including ICIs.

Serum cortisol levels were measured using the Elecsys ${ }^{\circledast}$ cortisol II assay kit (Roche Diagnostics), which exhibits cross-reactivity with 11-deoxycortisol (DOC) at 3.6\%. With the use of metyrapone, we cannot exclude the possibility that the increased DOC may affect the cortisol levels; however, in any case, the result of serum cortisol levels was very low.

Although there was a possibility that PD-1 inhibitor might cause hypophysitis that impairs ACTH secretion, this case showed a typical feature of ectopic ACTH syndrome caused by SCLC and ACTH, and cortisol levels were markedly elevated, in which pituitary ACTH secretion was considered to be suppressed. Therefore, it is suggested that pituitary ACTH did not play a role in this clinical course even if hypophysitis might have occurred. 
Interestingly, ACTH and cortisol levels transiently increased after the first and second chemotherapy (Fig. 1). Although the half-life of ACTH was short, it can be explained by the rapid release of intracellular ACTH caused by the strong tumour lysis effect. Tumour lysis syndrome was observed in some malignancies that showed hypersensitivity to chemotherapy, such as malignant lymphoma, but rare in SCLC (9).

EAS represents between 5 and 10\% of cases of ACTHdependent Cushing's syndrome (1). EAS results from unregulated ACTH expression by NETs, including SCLC, carcinoid, gut NET, and medullary thyroid carcinoma (7, 8). Recently, ICIs have been approved for SCLC, and clinical trials of ICIs for NETs have been performed (10). Although it depends on the tumours, induction of ICIs for the treatment of these diseases may change the pathological condition and care for paraneoplastic syndrome, including EAS.

In conclusion, we described a case of SCLC with EAS treated with atezolizumab plus conventional chemotherapy, showing adrenal insufficiency. Therefore, a close observation should be implemented on patients with adrenal insufficiency when treated with atezolizumab plus chemotherapy because of its high efficiency. Finally, the advancement in cancer therapy and the care for endocrine paraneoplastic syndrome needs to be adapted.

\section{Declaration of interest}

Y T received lecture fees from Novo Nordisk, Novartis, Eli Lilly, Recordati Rare Disease, Otsuka Pharma, and Ascendis Pharma and grant from Ono Pharma, Teijin Pharma, Kowa Pharma, Taisho Pharma, Daiichi Sankyo Pharma, and Tanabe Mitsubishi Pharma, and S M grant from Chugai Pharma and TAIHO Pharma.

\section{Funding}

This study was supported by the Ministry of Health, Labor and Welfare, Japan (Grants-in-Aid for Scientific Research on Hypothalamo-hypophyseal Disorders and Grants-in-Aid for Scientific Research on Endocrine Syndrome with Sexual Differentiation and Maturation to $Y$ T).

\section{Patient consent}

Written informed consent for publication of their clinical details and clinical images was obtained from the patient.

\section{Author contribution statement}

$\mathrm{H} \mathrm{N}$ contributed to patient care and wrote the case report. $\mathrm{Y} \mathrm{N}, \mathrm{H} \mathrm{K}, \mathrm{S} \mathrm{O}$, $\mathrm{T} \mathrm{M}$, and $\mathrm{Y} \mathrm{K}$ contributed to patient care and reviewed the manuscript. $\mathrm{Y} T$ contributed to patient care and reviewed the case report critically. $E \mathrm{H}$, $\mathrm{M} \mathrm{O}, \mathrm{SH}$, and $\mathrm{S} \mathrm{M}$ performed chemotherapy and reviewed the manuscripts. All authors approved the final manuscript for publication.

\section{References}

1 Zhou T, Wang Y, Zhao X, Liu Y, Wang YX, Gang XK \& Wang GX. Small cell lung cancer starting with diabetes mellitus: two case reports and literature review. World Journal of Clinical Cases 20197 1213-1220. (https://doi.org/10.12998/wjcc.v7.i10.1213)

2 Nagy-Mignotte H, Shestaeva O, Vignoud L, Guillem P, Ruckly S, Chabre O, Sakhri L, Duruisseaux M, Mousseau M, Timsit JF, et al. Prognostic impact of paraneoplastic Cushing's syndrome in small-cell lung cancer. Journal of Thoracic Oncology 20149 497-505. (https://doi. org/10.1097/JTO.0000000000000116)

3 Horn L, Mansfield AS, Szczęsna A, Havel L, Krzakowski M, Hochmair MJ, Huemer F, Losonczy G, Johnson ML, Nishio M, et al. First-line atezolizumab plus chemotherapy in extensive-stage smallcell lung cancer. New England Journal of Medicine 2018379 2220-2229. (https://doi.org/10.1056/NEJMoa1809064)

4 Suyama K, Naito Y, Yoh K, Niho S, Goto K, Ohmatsu H, Nishiwaki Y \& Ohe Y. Development of Cushing's syndrome during effective chemotherapy for small cell lung cancer. Internal Medicine 201150 335-338. (https://doi.org/10.2169/internalmedicine.50.4127)

5 Aoki M, Fujisaka Y, Tokioka S, Hirai A, Henmi Y, Inoue Y, Narabayashi K, Yamano T, Tamura Y, Egashira Y, et al. Small-cell lung cancer in a young adult nonsmoking patient with ectopic adrenocorticotropin (ACTH) production. Internal Medicine 201655 1337-1339. (https://doi.org/10.2169/internalmedicine.55.6139)

6 Agarwal KA \& Soe MH. Beyond the dual paraneoplastic syndromes of small-cell lung cancer with $\mathrm{ADH}$ and ACTH secretion: a case report with literature review and future implications. Case Reports in Oncological Medicine 20182018 4038397. (https://doi. org/10.1155/2018/4038397)

7 Lobo Ferreira T, Nunes da Silva T, Canário D \& Francisca Delerue M. Hypertension and severe hypokalaemia associated with ectopic ACTH production. BMJ Case Reports 20182018 bcr-2017. (https://doi. org/10.1136/bcr-2017-223406)

8 Jin T, Wu F, Sun SY, Zheng FP, Zhou JQ, Zhu YP \& Wang Z. Small cell lung cancer with panhypopituitarism due to ectopic adrenocorticotropic hormone syndrome: a case report. World Journal of Clinical Cases 20197 1177-1183. (https://doi.org/10.12998/wjcc. v7.i10.1177)

9 Mirrakhimov AE, Ali AM, Khan M \& Barbaryan A. Tumor lysis syndrome in solid tumors: an up to date review of the literature. Rare Tumors 20146 5389. (https://doi.org/10.4081/rt.2014.5389)

10 Weber MM \& Fottner C. Immune checkpoint inhibitors in the treatment of patients with neuroendocrine neoplasia. Oncology Research and Treatment 201841 306-312. (https://doi. org/10.1159/000488996)

Received in final form 11 August 2021

Accepted 6 September 2021 